\section{Conhecimento sobre asma das mães de crianças acometidas pela patologia, em área coberta pelo Programa Saúde da Família}

\author{
Mothers of children with asthma's \\ knowledge on the condition, in \\ area covered by the Family Health \\ Program
}

Ana Maria Siga Stephan' Juvenal Soares Dias da Costa ${ }^{\prime, 11}$

' Programa de Pós-Graduação em Saúde Coletiva da Universidade do Vale do Rio dos Sinos, São Leopoldo, RS.

" Departamento de Medicina Social, Faculdade de Medicina da Universidade Federal de Pelotas, Pelotas, RS.

\section{Resumo}

Objetivo: Avaliar o conhecimento sobre asma das mães de crianças com esta patologia e os fatores associados a este conhecimento. Método: Realizou-se um estudo censitário para identificar as crianças com asma utilizando-se o questionário do ISAAC (International Study of Asthma and Alergies in Childhood). As mães das crianças identificadas responderam a outro questionário que levantou dados demográficos, socioeconômicos, ambientais, biológicos, comportamentais, aspectos relacionados ao manejo da doença e utilização de serviços de saúde. Foi considerado como conhecimento o preenchimento de três critérios: manejo inicial adequado das exacerbações, reconhecimento do agravamento dos episódios de sibilância e dos fatores desencadeantes. Resultados: Das 258 mães entrevistadas, 67 (26,0\%) foram consideradas como tendo conhecimento adequado. $\mathrm{Na}$ análise dos fatores associados ao conhecimento materno, permaneceu associado ao desfecho, após ajuste: o sexo da criança, a prematuridade, a criança ter outras doenças atópicas e possuir medicação em casa para o manejo das exacerbações. Conclusões: O estudo mostrou que, apesar de relatarem ter recebido orientações, apenas um terço das mães efetuou mudanças ambientais e comportamentais. Além disso, quando se estabeleceu que o conhecimento adequado devesse ser o preenchimento dos três critérios, o número de mães classificadas foi ainda menor entre as respondentes. Estes achados reforçam a necessidade de intervenções educativas focadas nas deficiências do conhecimento, como meio de possibilitar às crianças asmáticas o desenvolvimento de atitudes de automanejo bem sucedidas.

Palavras-chave: Asma em crianças. Educação em saúde. Automanejo. Atenção primária à saúde. Estudos transversais. 


\section{Abstract}

Objective: To evaluate the knowledge that mothers of children with asthma have about the condition and the associated factors Methods: A survey was performed to identify children with asthma by applying the ISAAC questionnaire (International Study of Asthma and Allergies in Childhood). Mothers answered another questionnaire to collect demographic, socioeconomic, environmental, biological, and behavioral data and to identify factors associated with the management of the disease and the utilization of healthcare services. Meeting three criteria was considered as knowledge: appropriate initial management of an asthma episode, recognition of exacerbation, and recognition of triggering factors. Results: Of the 258 mothers interviewed, 67 (26.0\%) were classified as having appropriate knowledge. Adjusted statistical analysis revealed that the following factors were associated with outcome: the child's sex, prematurity, atopic co-morbidities, and availability of medication to manage an episode at home. Conclusions: This study showed that, although mothers reported having received orientation, only one third of them implemented environmental and behavioral changes. Moreover, when meeting three criteria was established as a requirement to define knowledge, this number was even lower among respondents. These findings strengthen the need to focus on educational interventions in knowledge deficiencies, to enable asthmatic children to develop successful self-management attitudes.

Keywords: Asthma in children. Health education. Self-management. Primary health care. Cross-sectional studies.

\section{Introdução}

No Brasil, a asma é responsável por cerca de $10 \%$ das internações hospitalares em crianças de 1 a 9 anos $^{1}$. É considerada a doença crônica mais comum na infância e uma das principais causas de consultas em serviços de emergência ${ }^{2,3}$ e absenteísmo escolar ${ }^{4}$.

O manejo da asma inclui a educação dos pacientes e familiares sobre a cronicidade da doença, a identificação dos fatores agravantes, o controle dos fatores desencadeantes e o uso adequado dos medicamentos 5 .

Os resultados finais de um estudo multicêntrico, realizado na última década em 56 países, apontaram para um aumento da prevalência de asma, principalmente em crianças e, apesar dos avanços no conhecimento da doença e no tratamento, a persistência de uma taxa alta de morbidade e mortalidade ${ }^{6,7}$.

Diversos estudos sustentam que as dificuldades no manejo da asma estão relacionadas à falta de programas educacionais $^{8,9}$. As orientações para a prevenção e tratamento dos sintomas são essenciais para a otimização do controle da doença. Os programas educacionais para pacientes com asma são custo-efetivos e, mesmo que haja aumento nos gastos com medicação e no número de consultas, isso é compensado pela redução de visitas aos serviços de emergência e de hospitalizações ${ }^{3}$.

O objetivo desse estudo foi avaliar o conhecimento materno no manejo inicial das exacerbações da doença, na prevenção dessas pelo reconhecimento dos fatores desencadeantes e percepção das situações em que os episódios da doença exigiam cuidados médicos.

\section{Metodologia}

Pelotas é um município com 323.158 habitantes, situado no Rio Grande do Sul. Conta com 56 unidades de atenção básica à saúde, entre as quais 17 são Unidades de Saúde da Família (USF). A USF Bom Jesus situa-se na zona urbana e atende 3.393 
famílias que apresentam baixa inserção socioeconômica.

Um estudo de base populacional realizado em pré-escolares de Pelotas, em 1998, mostrou uma prevalência de asma de $25,4 \%^{10}$. No Estado do Rio Grande do Sul e em Pelotas, as doenças do aparelho respiratório são as principais causas de hospitalização entre as crianças de 1 a 9 anos. Em Pelotas, durante o ano de 2008, a asma foi responsável por $20,1 \%$ das internações por doenças do aparelho respiratório nessa faixa etária ${ }^{11}$. Na ocasião em que este estudo foi realizado, o fornecimento de medicação para asma nas UBS era realizado de forma irregular e insuficiente.

Entre agosto e outubro de 2007 foi realizado um estudo transversal que incluiu todas as 1.165 crianças de 1 a 9 anos residentes na área de abrangência da USF. Os agentes de saúde foram treinados para aplicar o questionário do ISAAC ${ }^{4,13}$, que é um instrumento bastante sensível e específico, capaz de separar crianças asmáticas das não asmáticas ${ }^{14}$.

Para definição dos casos de asma adotaram-se os critérios do estudo de Ferrari et al. ${ }^{14}$ : crianças com 4 ou mais crises de sibilos no último ano, ou com 1 a 3 crises associadas à interrupçäo do sono por crises ou tosse noturna e sibilos após exercícios.

Dessa forma foram identificadas 258 crianças com asma atual, ou seja, presença de sintomas no último ano. As mães dessas crianças responderam a outro questionário que levantou dados demográficos, socioeconômicos, ambientais, biológicos, comportamentais, aspectos relacionados ao manejo da doença e utilização de serviços de saúde.

Para avaliação do conhecimento utilizaram-se questões abertas que foram póscodificadas.

O conhecimento materno foi avaliado através de três critérios, com o mesmo peso:

- Manejo inicial dos episódios de sibilância: para este critério foram consideradas as respostas a seguinte questão: "O que a senhora faz quando seu filho inicia uma crise de chiado no peito?”. A resposta foi classificada como adequada quando a mãe referia fazer uso de broncodilatador inalatório, oral ou em gotas para nebulização (de acordo com o III Consenso Brasileiro de Manejo de Asma $^{15} \mathrm{e}$ com a Global Initiative for Asthma-GINA ${ }^{5}$ ). Outras respostas, tais como esperar para ver se melhora e esperar para levar ao médico sem iniciar medicação, foram consideradas inadequadas.

- Reconhecimento de sinais e sintomas de gravidade da exacerbação: para este critério foi perguntado "Quando a criança está em crise, o que faz a senhora achar que deve levá-la à consulta imediatamente?" As respostas mais frequentes das mães foram colocadas no artigo de forma coloquial, com exceção de "ficar roxinho" que foi substituído pelo termo cianose. O reconhecimento do agravamento foi considerado adequado se a mãe referisse qualquer das seguintes situações: a criança não melhora com a medicação administrada, dificuldade para respirar, respiração curta, afundar embaixo das costelas ou no pescoço ao respirar, febre, cansaço para falar e cianose.

- Reconhecimento de fatores desencadeantes das exacerbações: foi solicitado que as mães escolhessem de uma lista de fatores (contato com mofo ou poeira domiciliar; contato com animais; presença de cheiros fortes; fumaça de cigarro; mudança de temperatura do ambiente; rir ou chorar demais; presença de resfriados; após exercício) aqueles que se relacionavam às exacerbações da doença do seu filho.

O desfecho, conhecimento adequado, foi considerado positivo quando os três critérios acima foram preenchidos.

Os dados foram digitados no programa Epi Info e a análise realizada nos programas SPSS 13.0 e Stata 9.0. As associações entre as variáveis e o desfecho foram investigadas através das razões de prevalência, com cálculo dos respectivos intervalos de confiança e análise da significância estatística utilizan- 
do o teste do Qui-quadrado de Pearson e de tendência linear.

A análise ajustada foi realizada utilizando-se regressão de Poisson com todas as variáveis entrando no modelo, independente do p-valor da análise bruta, conforme os níveis hierárquicos ${ }^{16}$ :

No nível mais distal: variáveis demográficas e socioeconômicas.

No segundo nível: variáveis relacionadas às morbidades, ambientais e comportamentais.

No nível mais proximal: variáveis relacionadas ao uso dos serviços de saúde.

Para manutenção no modelo de análise ajustada foram consideradas as variáveis com p-valor menor ou igual a 0,20 .

O controle de qualidade, em $10 \%$ da amostra, foi realizado por estudantes do $6^{\circ}$ semestre de Medicina, através de visitas domiciliares, pela reaplicação questionário sobre sintomas de asma, encontrando-se 86\% de concordância nas respostas.

O projeto foi aprovado pelo Comitê de Ética em Pesquisa da UNISINOS e a coleta de dados autorizada pela Secretaria Municipal de Saúde. As mães das crianças foram esclarecidas sobre o estudo e assinaram termo de consentimento.

\section{Resultados}

Entre as 1165 crianças da área de abrangência da USF, 1.140 foram encontradas, 19 mudaram de endereço e 6 recusaram-se a participar do estudo, totalizando $2,1 \%$ de perdas. A prevalência de asma atual encontrada foi de $22,6 \%$. A maioria das crianças incluídas no estudo encontrava-se na faixa etária de 1 a 6 anos (63,6\%), sendo que 51,4\% eram meninos. Em relação às características das mães $71,7 \%$ eram brancas, $52,8 \%$ tinham menos de 30 anos, $75,9 \%$ até 8 anos de escolaridade e $91,1 \%$ renda familiar abaixo de tres salários mínimos, ressaltando-se que metade das famílias possuía renda abaixo de um salário mínimo. Cerca de $11 \%$ das mães tinham mais de um filho com asma.

Quanto ao manejo inicial dos episódios de sibilância, 141 mães $(54,6 \%)$ apresenta- ram atitude adequada segundo os critérios do estudo. A maioria das mães $(62,8 \%)$ reconheceu ao menos um sintoma de agravamento da crise e todas citaram algum fator desencadeante, sendo que $50 \%$ delas apontaram até 4 fatores. (Tabela 1).

Cerca de 70\% (181) das mães admitiram ter recebido orientações e 44,6\% (115) fizeram modificações ambientais ou comportamentais. As orientações mais lembradas $(43,0 \%)$ foram relacionadas aos cuidados com poeira, mofo, cigarro e animais de estimação, e as modificações realizadas mais freqüentemente $(34,9 \%)$ foram retirar tapetes, cortinas, brinquedos de pelúcia e cuidados ao varrer e tirar o pó. A maioria das mães $(78,3 \%)$ tinha medicação adequada para tratamento inicial dos episódios de sibilância.

Entre as 258 mães entrevistadas, 67 $(26,0 \%)$ preencheram os três critérios e foram consideradas como tendo conhecimento adequado.

$\mathrm{Na}$ análise ajustada permaneceram associadas ao desfecho as variáveis: sexo da criança, prematuridade, criança com outras doenças atópicas e ter medicação para o manejo inicial das exacerbações da doença. (Tabela 2)

\section{Discussão}

Nesse estudo foram verificadas as atitudes das mães e criaram-se critérios que, se preenchidos, indicavam que as mães tinham conhecimento adequado sobre a doença.

A avaliação das questões relacionadas ao conhecimento das mães sobre asma mostrou que a maioria delas reconhecia os fatores desencadeantes das crises. O conhecimento é um conceito freqüentemente usado como preditor de comportamentos associados à promoção da saúde e ao desenvolvimento da capacidade de automanejo em doenças crônicas. A auto-eficácia está relacionada com a crença da pessoa na sua capacidade de exercer influência sobre as suas ações e associada à auto-estima e a outros fatores da história pessoal ${ }^{17}$. No pre- 
Tabela 1 - Frequências das respostas das mães de crianças com asma, moradoras na área de cobertura da USF Bom Jesus, às questões sobre o conhecimento da doença, Pelotas, 2007.

$\mathrm{N}=258$

Table 1 - Frequency of answers of mothers of children with asthma living in the catchment area of Bom Jesus FHU, to the questions on knowledge on the condition, Pelotas, 2007. N=258

\begin{tabular}{lcc}
\hline Variável & $\mathrm{N}$ & $\%$ \\
\hline Manejo inicial do episódio de sibilância & 44 & 17,1 \\
Aguarda a evolução do episódio & 73 & 28,3 \\
Leva ao médico sem iniciar medicação & 141 & 54,6 \\
Usa broncodilatador & & \\
& & \\
Reconhecimento de sinais e sintomas de gravidade & 30 & 11,6 \\
Não melhorar com a medicação & 184 & 71,3 \\
Dificuldade para respirar & 57 & 22,1 \\
Febre & 13 & 5,0 \\
Cansaço para falar & 14 & 5,4 \\
Cianose & & \\
& & \\
Reconhecimento de fatores desencadeantes & 130 & 50,4 \\
Mofo ou poeira & 60 & 23,3 \\
Pêlos de animais & 130 & 50,4 \\
Cheiros fortes & 130 & 50,4 \\
Fumaça de cigarro & 229 & 88,8 \\
Mudanças de temperatura & 103 & 39,9 \\
Estado emocional & 218 & 84,5 \\
Resfriados & 140 & 54,3 \\
Exercícios físicos &
\end{tabular}

Tabela 2 - Análise ajustada. Fatores associados ao conhecimento sobre asma em mães de crianças asmáticas, moradoras na área de cobertura da USF Bom Jesus, Pelotas, 2007. N = 258 Table 2 - Adjusted analysis. Factors associated with knowledge on asthma in mothers of asthmatic children, living in the catchment area of Bom Jesus FHU, Pelotas, 2007. N=258

\begin{tabular}{|c|c|c|c|}
\hline Nível $^{\mathrm{a}}$ & Variáveis & Razões de Prevalência(IC95\%) & Valor $\mathbf{p}^{b}$ \\
\hline$\overline{1}$ & Sexo & & $0,04^{*}$ \\
\hline & Masculino & 1,0 & \\
\hline & Feminino & $0,70(0,36-0,98)$ & \\
\hline & Cor da mãe & & $0,25^{*}$ \\
\hline & Não branca & 1,0 & \\
\hline & Branca & $1,42(0,78-2,58)$ & \\
\hline & Idade materna & & $0,25^{*}$ \\
\hline & Até 30 anos & 1,0 & \\
\hline & 31 anos ou mais & $1,33(0,81-2,20)$ & \\
\hline & Escolaridade da mãe & & $0,15^{*}$ \\
\hline & Até 8 anos de estudo & 1,0 & \\
\hline & Acima de 9 anos & $1,50(0,86-2,62)$ & \\
\hline & Renda familiar (SM) & & $0,11^{* *}$ \\
\hline & Abaixo de 1 & 1,0 & \\
\hline & 1 a 2,99 & $1,76(1,04-2,98)$ & \\
\hline & 3 ou mais & $1,44(0,59-3,48)$ & \\
\hline & №. de filhos & & $0,33^{*}$ \\
\hline & 4 ou mais & 1,0 & \\
\hline & Até 3 & $1,37(0,72-2,62)$ & \\
\hline
\end{tabular}


Tabela 2 - continuação

Table 2 - continuation

\begin{tabular}{|c|c|c|c|}
\hline Nívela $^{\mathrm{a}}$ & Variáveis & Razões de Prevalência(IC95\%) & Valor $\mathbf{p}^{\mathbf{b}}$ \\
\hline \multirow[t]{27}{*}{2} & Prematuridade & & $0,04^{*}$ \\
\hline & Não & 1,0 & \\
\hline & Sim & $1,80(1,03-3,15)$ & \\
\hline & História familiar de atopia & & $0,69^{*}$ \\
\hline & Não & 1,0 & \\
\hline & Sim & $1,10(0,67-1,85)$ & \\
\hline & Criança com outras atopias & & $0,05^{*}$ \\
\hline & Não & 1,0 & \\
\hline & Sim & $1,68(1,00-2,80)$ & \\
\hline & Internação ou sibilos no $1^{\circ}$ ano de & & $0,88^{*}$ \\
\hline & vida & 1,0 & \\
\hline & Não & $0,96(0,54-1,68)$ & \\
\hline & Sim & & $0,23^{* *}$ \\
\hline & Episódios de sibilância no último & 1,0 & \\
\hline & ano & $0,94(0,5-1,59)$ & \\
\hline & 1 a 3 & $1,87(0,83-4,20)$ & \\
\hline & 4 a 12 & & $0,15^{*}$ \\
\hline & Acima de 12 & 1,0 & \\
\hline & №. de moradores/peça & $1,69(0,83-3,45)$ & \\
\hline & 2 ou mais & & $0,10^{*}$ \\
\hline & Menos de 2 & 1,0 & \\
\hline & Fumantes no domicílio & $0,64(0,37-1,09)$ & \\
\hline & Sim & & $0,004^{*}$ \\
\hline & Não & 1,0 & \\
\hline & Ter medicação em casa & $5,60(1,75-17,95)$ & \\
\hline & Não & & \\
\hline & Sim & & \\
\hline
\end{tabular}

3 Recebeu orientações

Não

1,0

$0,73^{*}$

Sim

$1,15(0,52-2,52)$

Quem orientou

Não recebeu orientação

1,0

$0,26^{*}$

Médico do posto

Médico da emergência

$1,59(0,78-3,22)$

$1,05(0,48-2,33)$

Local onde consulta

Posto de Saúde

1,0

$0,58^{*}$

Pronto socorro

$0,92(0,42-1,99)$

Outro

$1,34(0,70-2,54)$

Consultas na emergência nos

últimos

6 meses

1,0

$0,46^{* *}$

$\begin{array}{ll}\text { Não } & 1,50(0,78-2,91) \\ 1 \text { vez } & 1,20(0,65-2,20) \\ 2 \text { ou mais } & \end{array}$

a:Todas as variáveis estão controladas para as demais do mesmo nível e para as dos níveis acima (com valor p entre 5 e $20 \%$ ). All variables are controlled for others of the same level and for greater levels (with p between 55 and 20\%)

b: Variáveis com nível de significância $>20 \%$ saíram do modelo. Variables whose levels of significance was $>20 \%$ were excluded from the model.

"Teste de heterogeneidade Heterogeneity test.

** Teste do qui-quadrado para tendência linear. Chi-square test for linear trend. 
sente estudo, apesar de relatar ter recebido orientações de fazê-las, apenas um terço das mães efetuou mudanças ambientais e comportamentais relacionadas a esse conhecimento. Além disso, quando se estabeleceu que o conhecimento adequado deveria ser o preenchimento dos três critérios, o número de mães classificadas foi ainda menor entre as respondentes. Sabe-se que mudanças ambientais podem ser difíceis de serem realizadas quando as condições socioeconômicas são desfavoráveis e, mesmo quando possíveis, dependem do entendimento do indivíduo sobre a necessidade de realizálas. Isso pode ser dito também a respeito de mudanças comportamentais que são fortemente ligadas à motivação pessoal e pressupõem a percepção da obtenção de benefício ${ }^{18}$.

Constatou-se que mais da metade das mães utilizava broncodilatador em casa no início da crise. Ter em casa a medicação para as crises foi uma condição que permaneceu associada ao conhecimento após a análise ajustada. Não se pode concluir se o maior conhecimento fez com que a mãe buscasse ter a medicação, ou se ter a medicação significava que a mãe foi orientada a usá-la e isso melhorou a habilidade de manejo das exacerbações. Conforme o Manual para Tratamento de Asma em Crianças da GINA ${ }^{5}$, a educação para o cuidado em asma deve envolver a criança e os familiares e incluir orientação para reconhecer os sinais e sintomas das exacerbações e o uso correto e precoce da medicação. Este estudo avaliou se a medicação era usada, não verificando se a técnica era adequada. Quase todas as pessoas com doenças crônicas podem desenvolver habilidades de automanejo, que são reforçadas positiva ou negativamente de acordo com o controle que o indivíduo consegue obter sobre a doença ${ }^{18}$. Segundo Bandura ${ }^{19}$, a auto-eficácia requer não apenas habilidades, mas também força de vontade em acreditar na capacidade de exercer uma determinada conduta, o que é um importante elo entre o saber e o fazer. A auto-eficácia resulta de um processo integrado do pensamento, afeto e motivação que possibilita a organização e a execução de ações para obtenção de resultados, mesmo face às dificuldades, e é um preditor da capacidade de perseverança na manutenção dos cuidados em pessoas com doenças crônicas, mais importante que o mero conhecimento das atitudes de automanejo.

A maioria das mães reconhecia como sintoma de gravidade a piora da dificuldade respiratória e levavam a criança à consulta. Neste estudo, entre os sinais e sintomas de gravidade, grande parte das mães referiu que se a criança, durante uma crise de asma, apresentasse febre, este seria um motivo para procurar atendimento médico. Optou-se por considerar adequada esta resposta, embora na literatura a febre não esteja relacionada como sinal de gravidade nos casos de exacerbação da doença, mas por ser indiscutível que, na presença deste sintoma, a criança deva ser avaliada pelo médico. Um ensaio clínico controlado e randomizado, cuja intervenção foi um programa educacional para o manejo de asma, avaliou vários parâmetros para verificar quais os mais sensíveis na avaliação dos resultados da intervenção. A conclusão foi que o relato dos sintomas feito pelos pais é um dos parâmetros mais sensíveis para avaliar o estado da doença e as mudanças que ocorreram após a intervenção ${ }^{20}$. Uma revisão da literatura avaliou intervenções educativas em asma, mostrando que estas podem produzir um efeito real benéfico sobre a doença e que provavelmente a educação praticada de modo simples e flexível, e de forma personalizada durante o atendimento, possa ter um efeito maior ou pelo menos igual ao das intervenções baseadas em técnicas educativas variadas e sofisticadas ${ }^{21}$.

Embora o estudo não tenha tido poder para demonstrar as associações, pode-se observar que essas apontavam na direção esperada, ou seja, as mães mais pobres, com baixa escolaridade, com famílias mais numerosas, e as mais jovens, possuíam menos conhecimento. Enquanto que a prevalência de maior conhecimento esteve associada às mães de crianças do sexo masculino, que 
foram prematuras, com outras doenças atópicas, com um número maior de crises anuais da doença, que levavam o filho para consultar no posto de saúde ou ambulatório e recebiam orientações do médico desses locais.

Diversos estudos mostraram que para se obter um bom controle da asma é importante o acompanhamento ambulatorial da criança, e que as crianças que consultam apenas durante as crises e em serviços de emergência, não obtêm um bom controle da doença ${ }^{2,3,10}$. Da mesma forma no presente estudo encontrou-se associação de uma prevalência maior de conhecimento materno adequado entre as mães que levavam o filho para consultar em ambulatório. Entretanto, deve-se ter cautela diante desse resultado, uma vez que esta variável não permaneceu associada na análise ajustada.

Um estudo realizado em Illinois, USA, indicou que crianças asmáticas, a partir dos 7 anos de idade, podem ser confiáveis informantes dos seus sintomas ${ }^{22}$. Para proporcionar uma vida normal às crianças com asma os cuidados aprendidos pelos pais devem ser transmitidos a elas e isso justifica a necessidade de verificar se o conhecimento desses é adequado, porque alguns pais podem ter um entendimento que gere condutas inadequadas no enfrentamento da doença ${ }^{23}$.

O presente estudo avaliou que, no local onde foi aplicado, a maioria das mães entrevistadas não possuíam conhecimento adequado sobre asma e este dado reforça a necessidade de intervenções educativas focadas nas deficiências de conhecimento, para possibilitar que as crianças asmáticas desenvolvam atitudes de automanejo bem sucedidas, aumentando a sua auto-eficácia e a capacidade de manutenção dos cuidados. Demonstrou ainda que, em áreas de cobertura da Estratégia Saúde da Família, podem ser realizados pequenos estudos transversais, rápidos e de baixo custo, treinando os agentes de saúde para a coleta de dados e, dessa forma, contribuir para melhorar a organização do trabalho das equipes.

\section{Referências}

1. Brasil. Ministério da Saúde, Sistema de Informações Hospitalares do SUS (SIH-SUS) 2001 [texto na Internet]. Brasília: Ministério da Saúde; 2002. Disponível em http://www.datasus.gov.br/catalogo/sihsus.htm. [Acessado em 20 de novembro de 2007]

2. Crain EF, Kercsmar C, Weiss KB, Mitchell H, Lynn $\mathrm{H}$. Reported difficulties in access to quality care for children with asthma in the inner-city. Arch Pediatr Adolesc Med 1998; 152: 333-9.

3. Friday GA, Khine H, Lin M, Caliguiri LA. Profile of children requiring emergency treatment for asthma. Ann Allergy Asthma Immunol 1997; 78: 221-4.

4. Fowler MG, Davenport MG, Garg R. School functioning of US children with asthma. Pediatrics 1992; 90: 939-44

5. Global Initiative for Asthma - GINA. Pocket Guide for Asthma management and Prevention in Children 2006. Avaliable in http:/ / www.ginasthma.com. Acessado em 05 de fevereiro de 2007.

6. International Study of Asthma and Allergies in Childhood (ISAAC): Worldwide variations in the prevalence of asthma symptoms. Eur Respir J 1998; 12(2): 315-35.
7. Asher MI; Keil U; Anderson HR; Beasley R; Crane J, Martinez F et al. Worldwide variation in prevalence of symptoms of asthma, allergic rhinoconjunctivitis, and atopic eczema: ISAAC (International Study of Asthma and Allergies in Childhood) Steering Committee. Lancet 1998; 351: 1225-32.

8. Emerson F; Tebyriça JN. Educação e Asma. Rev Bras de Alergia e Imunopatologia 1998; 21(6): 209-17.

9. Chiang LC, Huang JL, Yeh KW, Lu CM. Effects of a selfmanagement asthma educational program in Taiwan based on Precede-Proceed model for parents with asthmatic children. J Asthma 2004; 41(2): 205-15.

10. Chatkin M, Menezes AMB, Victora CG, Barros FC. Asthmatic children's risk factors for emergency room visits. Brazil. Rev Saúde Pública 2000; 34 (5): 491-8.

11. Brasil. Ministério da Saúde, Sistema de Informações Hospitalares do SUS (SIH-SUS) 2008. Disponível em: http://www.datasus.gov.br/catalogo/sihsus.htm. [Acessado em 07 de agosto de 2009]

12. University of Auckland. International Study of Asthma and Allergies in Childhood (ISAAC) Avaliable in http:// isaac.auckland.ac.nz [Último acesso em 10 de agosto de 2008] 
13. Solé D, Vanna AT, Yamada E, Rizzo MC, Naspitz CK. International study of asthma and allergies in childhood (ISAAC) written questionnaire: validation of the asthma component among Brazilian children. J Invest Allergol Clin Immunol 1998; 8: 376-82.

14. Ferrari FP, Rosário Filho NA, Ribas LFO, Callefe LG. Prevalência de Asma em Escolares de Curitiba - Projeto ISAAC. J Pediatr 1998; 74(4): 299-305.

15. Sociedade Brasileira de Alergia e Imunopatologia, Sociedade Brasileira de Pediatria, Sociedade Brasileira de Pneumologia e Tisiologia. III Consenso Brasileiro no Manejo de Asma. J Pneumol 2002; 28(S1).

16. Victora CG, Huttly SR, Fuchs SC, Olinto MTA. The role of conceptual frameworks in epidemiological analysis: a hierarchical approach. Int J Epidemiol 1997; 26: 224-2

17. Bandura A. Self-efficacy: Toward a unifying theory of behavioral change. Psychol Rev 1977; 84: 191-215.

18. Bandura A. Self-efficacy: The exercise of control. New York: W.H. Freeman; 1997.

19. Bandura A. Self-efficacy: The foundation of agency. In W. J. Perrig: Control of human behavior, mental processes, and consciousness. Erlbaum 2000; 17-33.
20. Sharek PJ, Mayer ML, Robinson TN, Shames RS, Umetsu DT, Bergmann DA. Agreement Among Measures of Asthma Status: A Prospective Study of Low-Income Children with Moderate to Severe Asthma. Pediatrics 2002; 110: 797-804.

21. Santana LF, Bastos RC, Martinez-Silveira MS, Freitas LS. Intervenções educativas em asma na infância: uma revisão analítica da literatura. J Bras Pneumol 2005; 31(5): 445-58.

22. Olson LM, Radecki L, Frintner MP, Weiss KB, Korfmacher J, Siegel RM. At What Age Can Children Report Dependably on Their Asthma Health Status? Pediatrics 2007; 119: 93-102.

23. Mansour ME, Lanphear BP, DeWit TG. Barriers to asthma care in urban children: Parent perspectives. Pediatrics 2000; 106: 512-520.

Recebido em: 06/05/09

Versão final reapresentada em: 24/08/09

Aprovado em: 31/08/09 